\title{
Actualización en el diagnóstico y manejo de la Infección Urinaria en pediatría.
}

\author{
PAULINA SALAS DEL C. ${ }^{1}$, PATRICIA BARRERA B. ${ }^{2}$, CLAUDIA GONZÁLEZ C. ${ }^{2}$, \\ PEDRO ZAMBRANO O. ${ }^{3}$, IGNACIO SALGADO D. ${ }^{1}$, LILY QUIROZ ${ }^{4}$, \\ ANA MARÍA LILLO D. ${ }^{5}$, PILAR HEVIA J. ${ }^{6}$, FELIPE CAVAGNARO SM. ${ }^{7}$ \\ 1. Nefrólogo Pediatra, Unidad de Nefrología, Hospital Exequiel González Cortés. \\ 2. Nefrólogo Pediatra, Unidad de Nefrología, Complejo Asistencial Dr. Sótero del Río. \\ 3 Nefrólogo Pediatra, División de Pediatría, Pontificia Universidad Católica de Chile. \\ 4. Nefrólogo Pediatra, Unidad de Nefrología, Hospital Roberto del Río. \\ 5. Nefrólogo Pediatra, Unidad de Nefrología, Hospital Luis Calvo Mackenna. \\ 6. Nefrólogo Pediatra, Unidad de Nefrología, Hospital San Juan de Dios. \\ 7. Nefrólogo Pediatra, Clínica Alemana, Universidad del Desarrollo. \\ RAMA DE NEFROLOGÍA. SOCIEDAD CHILENA DE PEDIATRÍA.
}

\begin{abstract}
\section{Update in urinary tract infection in pediatrics}

Urinary tract infection (UTI) is a common infectious disease in children. Traditionally UTI has been considered as a marker of underlying anatomical or functional urological abnormalities. In the last decades all children with febrile UTI were recommended to undergo imagenological studies looking for renal scarring or urinary tract abnormalities. The goal of this article it is to update the concepts of febrile UTI studies, treatments and follow-up. The imagenological studies are unpleasant for children and distressing for their parents. Current data do not provide enough evidence about the effectiveness of broad studies in UTI, therefore physicians should be more selective in order to find out patients who requiere surgical or medical intervention. These recommendations are based on NICE guidelines and support the concept that one of the most important issues to avoid renal damage is a prompt diagnosis and effective treatment.
\end{abstract}

(Key words: Urinary tract infection, renal abnormalities, renal scarring, urological malformation).

Rev Chil Pediatr 2012; 83 (3): 269-278

\section{RESUMEN}

La infección urinaria (ITU) es una de las patologías infecciosas más frecuentes en pediatría. Tradicionalmente se ha considerado como marcador de probables anormalidades anatómicas y funcionales de la vía urinaria, por lo cual, en las últimas décadas todos los niños que presentaban una ITU eran sometidos a estudio por imágenes en busca de cicatrices renales o anormalidades de la vía urinaria. El objetivo de este artículo es actualizar los conceptos sobre el estudio y tratamiento de pacientes pediátricos que presentan una ITU febril. El estudio por imágenes es incómodo para los pacientes, estresante para los padres y las evidencias actuales para continuar realizándolo son limitadas por lo cual debe ser selectivo en orden de focalizar en los pacientes que puedan 
beneficiarse con cirugías urológicas correctoras o tratamientos médicos adecuados. Estas pautas están basadas en las guías NICE de ITU e intentan demostrar que uno de los pilares más importantes para evitar secuelas renales es el diagnóstico y tratamiento oportuno de las ITU febriles.

(Palabras clave: Infección urinaria, malformación renal, cicatriz renal, malformación urológica).

Rev Chil Pediatr 2012; 83 (3): 269-278

Las presentes recomendaciones surgen de una revisión de las últimas publicaciones en relación a los avances en el diagnóstico y manejo de los niños con infección urinaria.

Estas recomendaciones están dirigidas a médicos generales, pediatras y nefrólogos infantiles, con el objetivo de facilitar las decisiones clínicas en el diagnóstico y tratamiento de las infecciones urinarias.(ITU).

Es importante considerar que estas guías deben adecuarse a la disponibilidad de recursos terapéuticos y tecnológicos locales. Además, cada lugar debe tener presente el patrón de infecciones, microorganismos involucrados y sensibilidad a antibióticos.

\section{Abreviaturas}

ITU infección urinaria.

HTA hipertensión arterial.

PNA pielonefritis aguda.

RVU reflujo vesico ureteral.

PE preeclamsia.

CR cicatriz renal.

ERC enfermedad renal crónica.

OC orina completa.

URC urocultivo.

UCG uretrocistografía.

CID cistografía isotópica directa.

RNM resonancia nuclear magnética.

TAC: tomografía axial computarizada.

\section{Definiciones}

La Infección del tracto urinario (ITU) se define clásicamente como la invasión, colonización y proliferación bacteriana del tracto urinario, que puede comprometer desde la vejiga hasta el parénquima renal.

La presentación clínica puede ser de 3 formas:
- Cistitis o ITU baja: Infección limitada a la vejiga y a la uretra, más frecuente en mujeres mayores de 2 años. Los pacientes refieren síntomas limitados a inflamación local como disuria, poliaquiuria, urgencia, orina turbia, y molestias abdominales bajas.

- Pielonefritis aguda o ITU alta: Infección que compromete el parénquima renal. Es la forma más grave de ITU en niños. Los pacientes generalmente presentan síntomas sistémicos como fiebre alta, compromiso del estado general, decaimiento, dolor abdominal, dolor lumbar y frecuentemente vómitos y mala tolerancia oral

Los 2 elementos clínicos que sugieren pielonefritis o ITU alta son fiebre y dolor lumbar.

En la literatura reciente se denomina ITU atípica a una ITU alta que evoluciona en forma tórpida. En este cuadro clínico, además de los síntomas descritos se pueden observar elementos que sugieren alteraciones anatómicas o funcionales de la vía urinaria tales como:

- Chorro urinario débil.

- Masa abdominal o vesical.

- Aumento de creatinina.

- Septicemia.

- Falla de respuesta al tratamiento antibiótico a las 48 horas.

- Infección por germen no E. coli.

- Bacteruria asintomática: Presencia de urocultivo positivo y ausencia de marcadores inflamatorios en el examen orina completo (OC) en pacientes sin sintomatología clínica. Habitualmente es un hallazgo en exámenes de orina tomados en seguimientos.

Se recomienda no indicar tratamiento antibiótico, ya que estudios a largo plazo no muestran beneficios en los grupos tratados.

- ITU recurrente definida como 3 o más ITU bajas, 2 o más pielonefritis o 1 pielonefritis más 1 ITU baja en un año. 


\section{Factores de riesgo asociados a ITU}

El diagnóstico y tratamiento precoz de las ITU ha demostrado ser determinante en evitar la aparición de cicatrices renales, por lo tanto, es importante identificar en la historia y en el examen físico factores de riesgo y elementos clínicos que sugieren una anormalidad de la vía urinaria que favorezca la primoinfección y la recurrencia de ITU.

También pueden aparecer signos clínicos que sugieren daño renal secundario a pielonefritis crónica como hipertensión arterial (HTA) y mal desarrollo pondoestatural.

De acuerdo a la literatura estos factores son:

- ITU previa.

- Historia de fiebre recurrente sin foco.

- Diagnóstico antenatal de anomalía renal.

- Antecedente familiar de reflujo vésico ureteral (RVU) o enfermedad renal.

- Constipación.

- Disfunción miccional.

- Chorro débil.

- Globo vesical.

- Masa abdominal.

- Lesión espinal.

- HTA.

- Mal desarrollo pondoestatural.

\section{Diagnóstico}

El diagnóstico de ITU debe plantearse frente a una historia y examen físico sugerente, asociado a un examen de orina compatible. Se confirma con un urocultivo positivo. El recuento de colonias significativo dependerá del método por el cual haya sido tomada la muestra.

En aquellos casos donde exista duda o discordancia entre estos elementos diagnósticos, los exámenes complementarios como el cintigrama renal con ácido dimercaptosuccínico (DMSA) o ecografía doppler renal pueden ser exámenes que ayudan a confirmar el diagnóstico.

Con respecto al método de recolección de orina es importante minimizar los riesgos de contaminación en la toma de muestra, por lo tanto, previamente debe realizarse un lavado de genitales con agua y jabón sin antisépticos.
La muestra debe ser tomada y sembrada de inmediato (orina fresca), si esto no es posible se mantendrá refrigerada a $4^{\circ} \mathrm{C}$ por un tiempo máximo de 24 horas. Es importante destacar que la muestra debe ser tomada por personal médico.

Existen métodos de recolección no invasivos como bolsa recolectora, recolección de segundo chorro y métodos invasivos como sondeo transuretral y punción vesical.

En niños sin control de esfínteres se recomienda el sondeo transuretral. De no ser posible debe realizarse una punción vesical, idealmente bajo visión ecográfica directa.

En niños mayores 2 años o continentes una muestra de orina obtenida por segundo chorro es adecuada.

Debido al alto índice de contaminación un urocultivo positivo obtenido por bolsa recolectora no se considera ITU. Se debe repetir la toma de muestra por sondeo o punción vesical para confirmar diagnóstico, previo a iniciar tratamiento antibiótico (tabla 1).

El urocultivo requiere mínimo $18 \mathrm{~h}$ de incubación para poder informar si hay crecimiento bacteriano, por lo tanto, es recomendable apoyar el diagnóstico en elementos del examen de orina validados como sugerentes de ITU y posteriormente confirmar con urocultivo. Para ello se requiere una muestra de orina para realizar estudio físico-químico y microscópico (tira reactiva más sedimento). Los indicadores de ITU en la tira reactiva son los leucocitos (leucocito esterasa) y los nitritos.

El examen de microscópico (sedimento urinario) se realizará con orina centrifugada con resultado por campo y orina sin centrifugar con resultado por microlitro. Son indicadores

\section{Tabla 1. Criterios microbiológicos de los diferentes métodos de recolección de orina en el diagnóstico de ITU en niños ${ }^{24}$}

\begin{tabular}{|lcc|}
\hline Método recolección & $\begin{array}{c}\text { n de } \\
\text { organismos }\end{array}$ & $\begin{array}{c}\text { n de colonias } \\
\text { por ml }\end{array}$ \\
\hline Punción suprapúbica & 1 & $>1$ \\
\hline Sondeo transuretral & 1 & $\geq 10000$ \\
Segundo chorro & 1 & $\geq 100000$ \\
Recolector & 1 & $\geq 100000$ \\
\hline
\end{tabular}


Tabla 2. Interpretación de resultados de tira reactiva y aproximación terapéutica (2)

\begin{tabular}{|cl|}
\hline Resultado tira reactiva & Conducta \\
\hline Leu (+) Nit (+) & Enviar muestra para urocultivo (URC) y examen de orina completa (OC) e iniciar antibiótico \\
Leu (-) Nit (+) & Si fue en orina fresca, enviar muestra para OC + URC e iniciar antibiótico \\
Leu (+) Nit (-) & $\begin{array}{l}\text { Si no existen síntomas específicos de ITU, no iniciar tratamiento hasta tener resultado de OC+ URC. } \\
\text { Considerar infección fuera del tracto urinario (infección genital) }\end{array}$ \\
\hline Leu (-) Nit (-) & $\begin{array}{l}\text { En paciente asintomático descarta ITU. En paciente sintomático tomar una muestra de OC + URC } \\
\text { para descartar ITU. Buscar otras patologías }\end{array}$ \\
\hline
\end{tabular}

Leu: leucocitos reactivos, Nit: nitritos, URC: urocultivo, OC: orina completa.

de infección urinaria la presencia de más de 5 leucocitos por campo en orina o más de 10 leucocitos por ul respectivamente. La bacteriuria no tiene punto de corte, en la mayoría de estudios es positiva con cualquier bacteria (1 bacteria por campo).

El examen microbiológico se puede realizar también en orina sin centrifugar a través de una tinción de Gram. La presencia de gérmenes sugiere ITU (tabla 2).

\section{Diagnóstico por imágenes}

\section{Objetivo}

Búsqueda de anomalías del tracto urinario que puedan predisponer al paciente a nuevas infecciones o a complicaciones de las mismas y que son susceptibles a tratamiento médico o quirúrgico.

\section{Tipos de estudios imagenológicos}

1. Ecografía renal con o sin doppler.

2. Cistografía:

- Uretrocistografía miccional (UCG).

- Cistografia isotopica directa (CID).

3. Cintigrama renal $\mathrm{TC}_{99}$-DMSA (ácido dimercaptosuccínico).

4. Otros.

\section{Propósitos}

I. Localización del nivel de la ITU.

II. Diagnóstico de RVU.

III.Diagnóstico de otras malformaciones del tracto urinario.

IV. Evaluación de secuelas: cicatriz renal (CR).

\section{Localización del nivel de ITU}

Sólo la ITU alta tiene riesgo de CR.

Los parámetros clínicos y de laboratorio carecen de la suficiente sensibilidad y especificidad para certificar el diagnóstico de pielonefritis aguda (PNA). La prevalencia de alteraciones del cintigrama renal con DMSA en la fase aguda va del 42 al $99 \%$ en diferentes estudios, dependiendo de los criterios de inclusión.

1. El cintigrama renal con DMSA en fase aguda (hasta 14 días desde el diagnóstico) es el gold standard de pielonefritis, pero su empleo rutinario no es viable en la mayoría de los centros.

2. La ecografía renal tiene un rendimiento muy variable dado la subjetividad de su interpretación. En general no es útil para diagnóstico de localización de la ITU, pero es inocua, está ampliamente disponible, y permite identificar anomalías urinarias preexistentes. Además podría modificar la terapia inicial con el hallazgo infrecuente de absceso renal.

3. La ecografía renal con power doppler permite evaluar la perfusión renal regional, que se encuentra disminuida en la infección aguda, teniendo buena correlación con el cintigrama con DMSA en la fase aguda.

4. Otros: resonancia nuclear magnética (RNM) requiere sedación, tomografía axial computarizada TAC produce alta dosis de radiación.

\section{Diagnóstico de RVU}

El RVU moderado y severo (III a V) se relaciona a ITU grave, ITU recurrente y mayor daño renal. Los RVU leves no se asocian con aumento de CR. 
1. La UCG es el examen de referencia para la detección del RVU. Es la única técnica que da información confiable de la uretra. Es muy simple de realizar en lactantes, pero puede tener complicaciones (infección, trauma uretral), irradia y genera stress en los niños y sus padres.

2. La CID tiene menor dosis de radiación y es más sensible que la UCG para detectar todos los grados de RVU (sensibilidad de $55 \%$ para UCG y $97 \%$ para CID). Es útil en el seguimiento de RVU diagnósticado por UCG convencional y podría utilizarse como método de diagnóstico de RVU en niñas sin anomalía anatómica evidente.

3. La ecografía convencional tiene rangos de sensibilidad y especificidad muy amplios y un cuociente de probabilidad cercano a 1, por lo que no es útil para diagnóstico de RVU.

4. La ecografía con contraste (ecocistografía) presenta mejor rendimiento diagnóstico que la ecografía convencional. Salvo en caso de sospecha de obstrucción uretral, podría reemplazar a la UCG, pero falta evaluar la reproducibilidad antes de generalizar la prueba.

\section{Diagnóstico de otras malformaciones del tracto urinario}

En presencia de obstrucción de la vía urinaria hay mayor frecuencia de enfermedad bacterémica y mayor daño renal.

Las anomalías estructurales más frecuentemente encontradas en niños que han tenido una ITU son dilataciones de la vía urinaria y riñón dúplex. La frecuencia va de 10 a $75 \%$ dependiendo de las características de los niños incluidos en cada estudio. Sólo un 1\% requiere corrección quirúrgica. A menor edad mayor detección de anomalías.

Con el desarrollo de la ecografía prenatal ha disminuido el número de lactantes y niños que tienen ITU asociada a malformación significativa del tracto urinario.

1. La ecografía renal constituye el examen de elección para identificar alteraciones anatómicas como anomalías renales de número o de posición, doble sistema excretor, quistes, litiasis y alteraciones vesicales.
En los países que disponen de políticas de salud con estudios ecográfícos de amplia cobertura y confiables para las embarazadas podrían obviar la ecografía renal frente a un primer episodio de ITU. Sin embargo, en nuestro medio la posibilidad de estudio prenatal es variable, por lo tanto, es necesario realizar la ecografía renal en pacientes con ITU para descartar malformaciones de la vía urinaria.

2. El TAC helicoidal con contraste y la RNM dan buena información anatómica de los uréteres y sistema colector. La RNM es útil también en uréteres ectópicos.

3. La pielografía intravenosa no está indicada en el estudio de rutina del niño con ITU, ya que ha sido reemplazada por la ecografía.

\section{Evaluación de secuelas: cicatriz renal}

La mayoría de las CR son leves o unilaterales. Sólo las CR severas o bilaterales se han asociado con desarrollo de HTA, mayor frecuencia de preeclamsia en el embarazo y enfermedad renal crónica (ERC).

El riesgo de desarrollar CR después de una ITU es variable dependiendo del grupo analizado (5-64\%). Tienen mayor riesgo de presentar CR los pacientes con:

- $1^{a}$ ITU febril en lactante menor.

- ITU recurrente.

- Presencia de RVU (especialmente grados moderados a severos).

- Germen no E. coli.

En niños está demostrado que el cintigrama renal con DMSA es la prueba de referencia para el diagnóstico de defectos parenquimatosos renales. Se recomienda realizar este examen entre 6-12 meses después del episodio de ITU y de acuerdo al informe tomar una conducta terapéutica.

Un cintigrama renal con DMSA normal descarta CR. Paciente puede ser dado de alta, salvo que presente factores de riesgo.

Un cintigrama renal con DMSA que informe defectos menores unilaterales. Paciente no requiere seguimiento, excepto si hay antece- 
dentes de ITU recurrente $\mathrm{u}$ otros factores de riesgo.

Un cintigrama renal con DMSA que muestra CR severas y bilaterales. Paciente deben ser controlado, más aun si presenta disfunción renal, HTA o proteinuria ya que debiera recibir terapias de renoprotección.

Cabe destacar que la presencia de alteraciones cintigráficas severas en fase aguda determina mayor riesgo de secuelas.

\section{Recomendaciones del estudio de imágenes en paciente con ITU}

El estudio con imágenes en niños con un primer episodio de ITU debe ser selectivo, ya que el estudio rutinario a todos los niños no ha demostrado efectividad clínica y tiene un costo elevado.

La estrategia de mayor utilidad para reducir la morbilidad de la ITU y prevenir las CR adquiridas es el diagnóstico oportuno y el tratamiento precoz de la ITU, principalmente en lactantes y niños pequeños.

El estudio completo ya sea precoz o diferido se debe focalizar en los grupos de riesgo de daño renal:

- Menores de 6 meses (mayor riesgo de malformaciones y obstrucción de la vía urinaria que niños mayores).

- ITU atípica (alto riesgo de defectos parenquimatosos renales significativos y anomalías estructurales significativas).

- ITU recurrente (la recurrencia de ITU se asocia a aumento progresivo del riesgo de daño renal).

A continuación se adjuntan las tablas con las indicaciones de estudio según edad y grupo de riesgo:

Niños de 0-12 meses

\begin{tabular}{|l|c|c|c|}
\hline $\begin{array}{l}\text { Niños de 0 a 12 } \\
\text { meses }\end{array}$ & $\begin{array}{c}\text { Buena } \\
\text { respuesta }\end{array}$ & $\begin{array}{c}\text { ITU grave } \\
\text { o atípica }\end{array}$ & $\begin{array}{c}\text { ITU } \\
\text { recurrente }\end{array}$ \\
\hline Ecografía precoz & No & Sí & Sí \\
\hline Ecografía diferida $^{a}$ & Sí & No & No \\
\hline $\begin{array}{l}\text { DMSA }^{\text {b } 6-12 ~ m e s e s ~} \\
\text { Post ITU febril }\end{array}$ & Sí & Sí & Sí \\
\hline$U_{C G} c$ & Sí & Sí & Sí \\
\hline
\end{tabular}

Niños de 1-3 años

\begin{tabular}{|c|c|c|c|}
\hline $\begin{array}{l}\text { Niños de } 1 \text { a } 3 \\
\text { años }\end{array}$ & $\begin{array}{c}\text { Buena } \\
\text { respuesta }\end{array}$ & $\begin{array}{l}\text { ITU grave } \\
\text { o atípica }\end{array}$ & $\begin{array}{c}\text { ITU } \\
\text { recurrente }\end{array}$ \\
\hline Ecografía precoz & No & Sí & No \\
\hline Ecografía diferidaa & Sí & No & $S_{i}^{d}$ \\
\hline $\begin{array}{l}\text { DMSA }^{b} 6-12 \text { meses } \\
\text { Post ITU febril }\end{array}$ & $\mathrm{Si}$ & Sí & Sí \\
\hline UCGc & Selectiva ${ }^{e}$ & Si & Si \\
\hline
\end{tabular}

Niños $>3$ años

\begin{tabular}{|l|c|c|c|}
\hline $\begin{array}{l}\text { Niños mayor de } \\
\mathbf{3} \text { años }\end{array}$ & $\begin{array}{c}\text { Buena } \\
\text { respuesta }\end{array}$ & $\begin{array}{c}\text { ITU grave } \\
\text { o atípica }\end{array}$ & $\begin{array}{c}\text { ITU } \\
\text { recurrente }\end{array}$ \\
\hline Ecografía precoz & No & Síf $^{\prime f}$ & No \\
\hline Ecografía diferida & Síf $^{\prime}$ & No & Síd,f \\
\hline $\begin{array}{l}\text { DMSA } \\
\text { Post ITU 6-12 meses }\end{array}$ & Opcional & Si & Sí \\
\hline UCGc & Selectiva & Selectiva & Selectiva \\
\hline
\end{tabular}

a Realizar la ecografía dentro de las 6 semanas siguientes a la ITU. 'bE cintigrama renal con DMSA en fase aguda (antes de 2 semanas), permite orientar el seguimiento y realización o no de otras pruebas. Si es normal no debe repetirse a los 6 meses. 'Al realizar UCG, administrar profilaxis antibiótica 3 días y realizar la prueba el $2^{\circ}$ día de profilaxis. ${ }^{d}$ No se recomienda repetirla en nuevos episodios de ITU, salvo en sospecha de síndrome de eliminación disfuncional. eRealizar UCG si en caso de ITU atípica, antecedentes familiares de RVU, dilatación de vías urinarias en la ecografía renal, cintigrama con DMSA alterado. 'La ecografía en los niños con control de esfínteres debe ser con vejiga llena, midiendo el volumen vesical antes y después de una micción. gFrente a la sospecha de disfunción vesical y/o esfinteriana asociada a ITU realizar una UCG (incluyendo placas postmiccionales) y evaluar necesidad de estudio urodinámico

Al resumir las recomendaciones de estudio por imágenes considerando las publicaciones internacionales recientes y la realidad local, las indicaciones ideales serían las siguientes:

\section{Ecografia renal}

Todos los paciente con ITU, independiente de localización y edad.

\section{Uretrocistografía Miccional}

Menor de un año.

ITU atípica.

Antecedentes de familiares directos con RVU.

Dilatación de vías urinarias en la ecografía renal.

Cintigrama con DMSA alterado. 


\section{Cintigrama renal DMSA}

Fase aguda (si existe disponibilidad): ITU febril e ITU dudosa.

Fase tardía: Toda ITU febril entre 6-12 meses posterior al episodio.

\section{Cistografía isotópica directa}

Control de RVU.

ITU febril recurrente con UCG normal.

\section{Propuesta alternativa (basada en cintigrama con DMSA)}

Frente a un primer episodio de ITU febril (independientemente de la edad), realizar ecografía renal-vesical $(R V)$ y cintigrama con DMSA en fase aguda.

Si Ecografía RV y cintigrama con DMSA son normales, no realizar UCG.

Si Ecografía RV y/o cintigrama con DMSA muestra alteraciones, completar estudio con UCG.

\section{Tratamiento}

Los objetivos de la terapia de la infección urinaria son:

- Obtener la mejoría clínica.

- Evitar la diseminación de la infección.

- Evitar complicaciones a largo plazo.

\section{Medidas generales}

Hidratación adecuada.

Educar sobre hábitos miccionales: Micción cada 3 hrs, no posponer deseo miccional.

Educar sobre hábitos defecatorios: Evitar constipación, aseo adecuado.

\section{Tratamiento antibiótico}

La elección del antibiótico depende de los gérmenes más habituales y del patrón de resistencia local. Las unidades de microbiología de cada establecimiento debieran mantener un registro constante, tanto de los gérmenes más frecuentes encontrados en su comunidad, como el patrón de resistencia de éstos y tener esta información disponible para los clínicos.

En líneas generales se recomienda:

\section{ITU baja}

En ITU baja sin antecedentes de patología conocida de la vía urinaria, la evidencia actual se inclina hacia el tratamiento acortado de 3-4 días versus el tratamiento estándar de 7 días.

Si el paciente recibía profilaxis, debe iniciar tratamiento con otro antibiótico.

Primera línea: Pacientes de 1-4 meses tratamiento oral con cefalosporinas.

En mayor de 4 meses nitrofurantoína, cotrimoxazol, cefalosporinas.

Segunda línea: quinolonas.

\section{ITU alta}

Lactantes mayores de 3 meses con buen estado general, sin aspecto tóxico y con posibilidad de ser evaluado en las 48 hrs siguientes pueden recibir terapia oral antibiótica desde el inicio

La terapia antibiótica endovenosa y la hospitalización queda reservada para aquellos con:

- Edad menor a 3 meses.

- Sepsis clínica o potencial bacteremia.

- Inmunosupresión.

- Vómitos o incapacidad de tolerar medicamento oral.

- Falta de adecuado control ambulatorio.

- Falta de respuesta a terapia ambulatoria.

Duración del tratamiento: 7- 10 días. En recién nacido 10-14 días.

Primera línea: Lactantes menores de 3 meses requiere tratamiento empírico endovenoso con ampicilina asociados a aminoglicósido o cefalosporina de $3^{\circ}$ generación, con el objetivo de cubrir gérmenes causantes de sepsis neonatal. Una vez confirmado el diagnóstico de ITU se continúa sólo con cefalosporina.

En mayor de 3 meses cefalosporinas.

Segunda línea: aminoglucósidos o quinolonas.

Si el paciente debe recibir aminoglicósidos, se recomienda evaluar función renal e indicar antibiótico en una dosis diaria de preferencia por vía endovenosa.

Se debe insistir en observar la evolución clínica a las $48 \mathrm{hrs}$. Sugiere una buena respuesta al tratamiento la mejoría clínica a las $48 \mathrm{hrs}$ y el urocultivo positivo a $E$. coli. 
Se define como mala respuesta al tratamiento la mantención de los síntomas o el mayor compromiso del estado general a las 48 hrs. En este caso sospechar ITU atípica y realizar OC+URC de control y estudio de imágenes en forma precoz (tabla 3 ).

\section{Profilaxis antibiótica}

En la actualidad las indicaciones de profilaxis antibiotica para prevenir ITU han disminuido significativamente. Este cambio de conducta está basado en estudios controlados y aleatorizados que han demostrado que la profilaxis no disminuye el riesgo de recurrencia de ITU febril 12 meses después del primer episodio en niños con o sin reflujo ${ }^{33-36}$.

Se aconsejan utilizar profilaxis sólo en las siguientes situaciones:

- Diagnóstico antenatal de anomalía vía urinaria mientras completa estudio.

- Menor de 2 años con ITU febril, hasta completar estudio de imágenes.

- RVU GIII o mayor, ya que pacientes con grados menores de reflujo tienen baja posibilidad de presentar nueva ITU febril.

- ITU recurrente.

- Disfunción vesical, mientras mejora patrón miccional.

\section{Seguimiento}

Todo paciente tratado por ITU debe realizarse un OC+URO una vez terminado el tratamiento con el objetivo de confirmar la desaparición de microorganismos en la vía urinaria. Sin embargo, está demostrado que continuar el estudio y seguimiento con exámenes de orina de rutina no permite anticipar el diagnóstico de ITU.

Actualmente se sugiere educar a los padres o cuidadores sobre los síntomas y signos de ITU, entregarles esta información por escrito y evaluar que tengan acceso a un centro médico para que los pacientes puedan realizarse precozmente un OC + URO e iniciar tratamiento antibiótico si está indicado.

Los pacientes con antecedentes de un epi-
Tabla 3. Dosis de antibióticos para tratamiento de ITU

\begin{tabular}{|c|c|c|c|}
\hline Nombre & $\begin{array}{c}\text { Dosis } \\
\mathbf{m g} / \mathbf{k g} / \text { día }\end{array}$ & $\begin{array}{l}\text { Fracciona- } \\
\text { miento }\end{array}$ & vía \\
\hline Nitrofurantoína & $5-7$ & Cada $8-12 \mathrm{hr}$ & Oral \\
\hline $\begin{array}{l}\text { Cotrimoxazol } \\
\text { (sulfa/TMP) }\end{array}$ & $40 / 7$ & Cada $12 \mathrm{hr}$ & Oral \\
\hline Cefadroxilo & 50 & Cada 8 - 12 hr & Oral \\
\hline Cefiximo & 8 & Cada $24 \mathrm{hr}$ & Oral \\
\hline Cefuroximo & 30 & Cada $12 \mathrm{hr}$ & Oral \\
\hline Cefpodoximo & 10 & Cada $12 \mathrm{hr}$ & Oral \\
\hline Cefotaxima & $100-150$ & Cada 6-8 hrs & e.v \\
\hline Ceftriaxone & 100 & Cada 24 hrs & e.v \\
\hline Amikacina & 15 & Cada 24 hr & e.vo i.m \\
\hline Gentamicina & 5 & Cada $24 \mathrm{hr}$ & e.vo i.m \\
\hline Ciprofloxacino & $20-30$ & Cada $24 \mathrm{hr}$ & Oral, e.v \\
\hline
\end{tabular}

sodio de ITU alta cuyo estudio por imágenes resultó normal o cintigrama DMSA sólo mostró defectos parenquimatosos renales leves, unilaterales no requieren seguimiento, excepto si presentan factores de riesgo o existen antecedentes familiares de ITU recurrente o HTA crónica.

Los pacientes con ITU recurrente deben ser evaluados por especialista para decidir estudio y tratamiento a seguir.

Los pacientes con cicatrices renales, más aun, si estas son bilaterales deben controlarse rutinariamente para evaluar peso, talla, HTA, proteinuria y función renal e iniciar terapias de renoprotección en el momento que lo requiera.

\section{Referencias}

1.- Palazzi D, Campbell J: Acute cystitis in children older than two years and adolescentes. UpToDate 2010.

2.- National Collaborating Centre for Women's and Children's Health: National Institute for Health and Clinical excellence (NICE). Urinary tract infection: Diagnosis, treatment and longterm management of urinary tract infection in children. Draft for consultation (October 2006). Accesible en línea en http://guidance. nice.org.uk/CG54.

3.- Wolff $M$ : Antimicrobianos para el médico general en el Chile del nuevo siglo. Rev Chil Infect 2002; 19 (Supl 


\section{3): S197-S204.}

4.- Cortés M, Salazar A, Acuña J: Cambios en la sensibilidad antibiótica de E. coli en mujeres con infección urinaria baja ambulatoria en un período de 10 años. Rev Chil Urol 2007; 72 (3): 289-91.

5.- Chang S, Shortliffe L: Pediatric Urinary Tract Infections. Pediatr Clin N Am 2006; (53): 379-400.

6.- Shaik N, Hoberman A: Acute management, imaging, and prognosis of urinary tract infection in children. UpToDate 2010.

7.- Chalumeau $M$, et al: Fluoroquinolone Safety in Pediatric Patients: A Prospective, Multicenter Comparative Cohort Study in France. Pediatrics 2007; 111: e714-9.

8.- Michael M, Hodson EM, Craig JC: Tratamiento antibiótico de corta duración versus estándar para la infección urinaria aguda en niños. Biblioteca Cochrane Plus 2008, $\mathrm{N}^{\circ} 2$.

9.- Dairiki L: Infection and inflammation of the Pediatric genitourinary tract. Wein: Campbell-Walsh Urology, 9th ed, 2007.

10.- Ochoa C, Málaga S: Recomendaciones de la Conferencia de Consenso "Manejo Diagnóstico y Terapéutico de las Infecciones del Tracto Urinario en la Infancia. An Pediatr (Barc) 200; 767 (5): 517-25.

11.- Salas P, Álvarez E, Saieh C: Pautas de diagnóstico y tratamiento en infección urinaria en niños. Documento de la Rama de Nefrología de la Sociedad Chilena de Pediatría. Rev Chil Pediatr 2003; 74 (3): 311-4.

12.- Kass EH: Bacteriuria and the diagnosis of infections of the urinary tract; with observations on the use of methionine as a urinary antiseptic. AMA Arch Intern Med 1957; 100 (5): 709-14.

13.- Hellerstein S, Duggan E, Welchert E, Grossman H, Sharma P: Localization of the site of urinary tract infections with the bladder washout test. J Pediatr 1981; 98 (2): 201-6.

14.- Hoberman A, Wald ER, Reynolds EA, Penchansky L, Charron M: Pyuria and bacteriuria in urine specimens obtained by catheter from young children with fever. J Pediatr 1994; 124 (4): 513-9.

15.- Shaikh N, Morone NE, Bost JE, Farrell MH: Prevalence of urinary tract infection in childhood: a meta-analysis. Pediatr Infect Dis J 2008; 27 (4): 302-8.

16.- Shaikh N, Morone NE, López J, et al: Does this child have a urinary tract infection? JAMA 2007; 298 (24): 2895-904.

17.- Gorelick MH, Hoberman A, Kearney D, Wald E, Shaw $K N$ : Validation of a decision rule identifying febrile young girls at high risk for urinary tract infection. Pe- diatr Emerg Care 2003; 19 (3): 162-4.

18.- Jodal $U$ : The natural history of bacteriuria in childhood. Infect Dis Clin North Am 1987; 1 (4): 713-29.

19.- Hoberman A, Wald ER, Hickey RW, et al: Oral versus initial intravenous therapy forurinary tract infections in young febrile children. Pediatrics 1999; 104 (1 Pt1): 79-86.

20.- Montini G, Toffolo A, Zucchetta P, et al: Antibiotic treatment for pyelonephritis in children: multicentre randomised controlled non-inferiority trial. BMJ 2007; 335 (7616): 386.

21.- Neuhaus TJ, Berger C, Buechner K, et al: Randomised trial of oral versus sequential intravenous/oral cephalosporins in children with pyelonephritis. Eur J Pediatr. 2008; 167 (9): 1037-47. Epub 2007 Dec 12.

22.- Hodson EM, Willis NS, Craig JC: Antibiotics for acute pyelonephritis in children. Cochrane Database Syst Rev 2007; (4): CD003772.

23.- Mori R, Lakhanpaul M, Verrier-Jones K: Diagnosis and management of urinary tract infection in children: summary of NICE guidance. BMJ 2007; 335 (7616): 395-7.

24.- Geary D, Schaefer F: Comprenhensive Pediatric Nephrology. Cap 35, pag 539-48. 2008.

25.- Ochoa C, Sangrador E, Rodríguez F y Grupo Investigador del Proyecto: Pruebas diagnósticas de imagen recomendadas en la infección urinaria. An Pediatr (Barc) 2007; 67 (5): 498-516.

26.- Urinary tract infection in children: diagnosis, treatment and long-term management. National Collaborating Centre for Women's and Children's Health Commissioned by the National Institute for Health and Clinical Excellence. August 2007.

27.- Ochoa Sangrador C, Málaga Guerrero S, Panel de expertos de la Conferencia de Consenso y Grupo Investigador de la Conferencia de Consenso: Recomendaciones de la Conferencia de Consenso "manejo diagnóstico y terapéutico de las infecciones del tracto urinario en la infancia”. An Pediatr (Barc) 2007; 67 (5): 517-25.

28.- UTI Guideline Team, Cincinnati Children's Hospital Medical Center: Evidence-based care guideline for medical management of first urinary tract infection in children 12 years of age or less, http://www.cincinnatichildrens.org/svc/dept-div/health-policy/ev-based/uti. htm, Guideline 7, pages 1-23, November, 2006.

29.- Value of Imaging Studies after a First Febrile Urinary Tract Infection in Young Children: Data from Italian Renal Infection Study 1. Montini et al. Pediatrics 2009; 123: e239-e246 http://www.pediatrics.org/cgi/content/ full/123/2/e239. 
30.- Urinary tract infection guideline of the Royal Children's Hospital Melbourne. 2009. http://www.rch.org.au/clinicalguide/cpg.cfm?doc_id=5241.

31.- Pohl H, Belman A B: The "top-down" approach to the evaluation of children with febrile urinary tract infection. Advances in Urology 2009, doi: 10.1155/ 2009/783409.

32.- Biassoni L, Chippington S: Imaging in Urinary Tract Infections: Current Strategies and New Trends. Semin Nucl Med 2008; 38: 56-66.

33.- Montini G: Urinary tract infections: to prophylaxis or not to prophylaxis. Pediatr Nephrol 2009; 1605-9.

34.- Roussey-Kesler: Antibiotic prophylaxis for the prevention of recurrent urinary tract infection in children with low-grade vesicoureteral reflux: results from a prospec- tive randomized study. J Urol 2008; 179: 674-9.

35.- Pennesi M, North East Italy Prophylaxis in VUR study group: Is antibiotic prophylaxis in children with vesicoureteral reflux effective in preventing pyelonephritis and renal scars? A randomized, controlled trial. Pediatrics 2008; 121: e1489-94.

36.- Montini G, IRIS Group: Prophylaxis after first febrile urinary tract infection in children? A multicenter, randomized, controlled non inferiority trial. Pediatrics 2008; 122: 1064-71.

37.- Garín E, Olavarría F, García Nieto V, et al: Clinical significance of primary vesicoureteral reflux and urinary antibiotic prophylaxis after acute pyelonephritis: a multicenter, randomized, controlled study. Pediatrics 2006; 117: 626-32. 